regions. In patients with insidious neurological progression for 1 year, evidence for DIS may be based on 1 or more T2 lesions in periventricular, juxtacortical or infratentorial regions or spinal cord, and/or positive oligoclonal bands in CSF. (Polman $\mathrm{CH}$, Reingold $\mathrm{SC}$, Banwell B, et al. Diagnostic criteria for multiple sclerosis. 2010 revisions to the McDonald criteria. Ann Neurol 2011 Feb;69(2):292-302).

These revisions are meant to simplify the diagnostic criteria, preserve their sensitivity and specificity, address their use across populations, and may allow earlier diagnosis. They accurately identify children older than 11 years at risk for MS relapses, but they are not ideal for younger children and are not suited for those with ADEM-like presentations.

\title{
VITAMIN D AND NEW MRI LESIONS IN MULTIPLE SCLEROSIS
}

Researchers from the University of California at San Francisco and from other centers in the US and Italy determined whether vitamin D status is associated with the development of new T2 lesions or contrast-enhancing lesions on brain MRI in patients with relapsing MS. Participants with MS (aged 18-70) were recruited for the study primarily from the UCSF MS Center. A diagnosis of MS or clinically isolated syndrome was made using the International Panel criteria. In a total of 2,362 3T brain MRI scans acquired from 469 subjects, each $10 \mathrm{ng} / \mathrm{ml}$ higher 25 -hydroxyvitamin $\mathrm{D}$ level was associated with a $15 \%$ lower risk of a new T2 lesion $(\mathrm{p}=0.004)$, a $32 \%$ lower risk of a gadolinium-enhancing lesion $(p=0.002)$, and lower subsequent disability $(p=0.037)$. Higher vitamin D levels were associated with a trend toward lower risk of relapse. Vitamin D levels are inversely associated with MS activity on brain MRI. Active smokers were at substantially greater risk of developing T2 lesions and clinical relapse. (Mowry EM, Waubant E, McCulloch CE, et al. Vitamin D status predicts new brain magnetic resonance imaging activity in multiple sclerosis. Ann Neurol 2012 Aug;72(2):234-40). (Multiple Sclerosis Center, Department of Neurology, University of California at San Francisco, San Francisco, CA. Respond: Dr EM Mowry. E-mail: emowry1@jhmi.edu).

COMMENT. MS patients in the study have begun supplementation with vitamin $\mathrm{D}$, and a randomized trial is recommended to establish clinical efficacy. The authors emphasize that observational studies alone can lead to incorrect conclusions. An overview of the literature on the nutritional factors most commonly implicated as having an effect on MS and the biological rationale for their use is provided (von Geldern $\mathrm{G}$, Mowry EM. The influence of nutritional factors on the prognosis of multiple sclerosis. Nat Rev Neurol 2012 Oct 2. [Epub ahead of print]). These include polyunsaturated fatty acids (PUFAs), milk proteins, probiotics, antioxidants, and Ginkgo biloba extracts. Further studies are needed to evaluate the effects of these dietary components on the relapse rate and progression of MS.

In contrast to the above emphasis on the positive role of vitamin D in MS, one authority finds that MS prevention does not require intake of more vitamin D and calcium than currently recommended for optimal bone health. (Peterlik M. Vitamin D insufficiency and chronic diseases: hype and reality. Food Funct 2012 Aug;3(8):784-94). 